Revue des patrimoines

37 | 2018

Jardins collectifs : de l'abbé Lemire aux jardins d'insertion. Typologies - Expériences - Enjeux de conservation

\title{
Jardins interculturels : regards sur un projet transculturel innovant en Allemagne
}

Intercultural gardens, an innovative transcultural project in Germany

\section{Christa Müller}

\section{OpenEdition}

Journals

Édition électronique

URL : http://journals.openedition.org/insitu/19152

DOI : 10.4000/insitu.19152

ISSN : $1630-7305$

Éditeur

Ministère de la culture

Référence électronique

Christa Müller, « Jardins interculturels : regards sur un projet transculturel innovant en Allemagne », In Situ [En ligne], 37 | 2018, mis en ligne le 17 décembre 2018, consulté le 30 avril 2019. URL : http:// journals.openedition.org/insitu/19152 ; DOI : 10.4000/insitu.19152

Ce document a été généré automatiquement le 30 avril 2019.

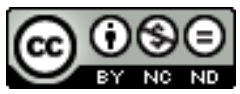

In Situ Revues des patrimoines est mis à disposition selon les termes de la licence Creative Commons Attribution - Pas d'Utilisation Commerciale - Pas de Modification 4.0 International. 


\title{
Jardins interculturels : regards sur un projet transculturel innovant en Allemagne
}

\author{
Intercultural gardens, an innovative transcultural project in Germany
}

Christa Müller

Merci à Catherine Gros, Hélia Paukner, Laurence Baudelet et Kerstin Stelmacher d'avoir assuré la traduction du texte dans la subtilité des concepts couverts par ce sujet.

Le mouvement d'exode déclenché par la guerre civile en Syrie a ravivé le débat public sur le concept, toujours flou, de "culture dominante $»^{1}$. Comme on l'a vu dans les récents affrontements entre partisans du multiculturalisme et tenants d'une exigence stricte $d^{\prime}$ '« assimilation » des immigrés dans une société d'accueil ${ }^{2}$ que l'on imagine statique, on constate actuellement une aggravation de ce dualisme. Mais on exclut souvent de ces discussions les réalités concrètes de la vie des migrantes et migrants. Dès le mouvement d'exil entraîné par la guerre de Bosnie, au milieu des années 1990, est né un projet innovant qui a expérimenté une démarche constructive vis-à-vis des différences culturelles, et que l'on peut voir comme une réponse pratique aux exacerbations des tensions sous-jacentes. Dans le projet des jardins interculturels, le fait de jardiner ensemble donne naissance à des processus transculturels de rencontre et d'échange. Dans ces jardins collectifs, on utilise les ressources qu'apportent de leurs pays d'origine ces hommes et ces femmes comme point de départ d'un processus d'autonomisation, et ce, de façon constructive. Cette contribution montre le développement des jardins interculturels depuis 1996 à travers l'exemple des « Jardins Internationaux de Göttingen » et traite des différentes potentialités de cette innovation sociale.

\section{Histoire des Jardins Interculturels en Allemagne}

2 Avec les Jardins Interculturels s'est constituée à partir du vécu des migrants une approche très riche qui permet de répondre de façon sociale, productive et créative au 
risque d'exclusion sociale. Dans les jardins interculturels, des hommes et des femmes issus de l'immigration, et des Allemands de souche appartenant à divers milieux sociaux, cultivent ensemble des fruits et des légumes, échangent des semences et discutent des modes de préparation du sol, pratiquent l'apiculture, construisent des fours à pain en terre et développent des concepts d'éducation à l'environnement qui incluent les savoirfaire issus des différentes cultures. Mais avant tout, ils produisent de la reconnaissance mutuelle.

3 Le dessin d'un Jardin Interculturel type comporte un espace commun, où l'on peut manger ensemble, faire des fêtes et où les enfants jouent (fig. 1). Il occupe en moyenne un tiers de la superficie totale. Et pour des jardinières et jardiniers qui vivent dans des appartements exigus, cet espace de convivialité fait souvent office de pièce de vie supplémentaire, en plein air. Sur les deux autres tiers de la surface, des parcelles de 10 à $100 \mathrm{~m}^{2}$, sans clôtures, sont consacrées à la culture des légumes.

Figure 1

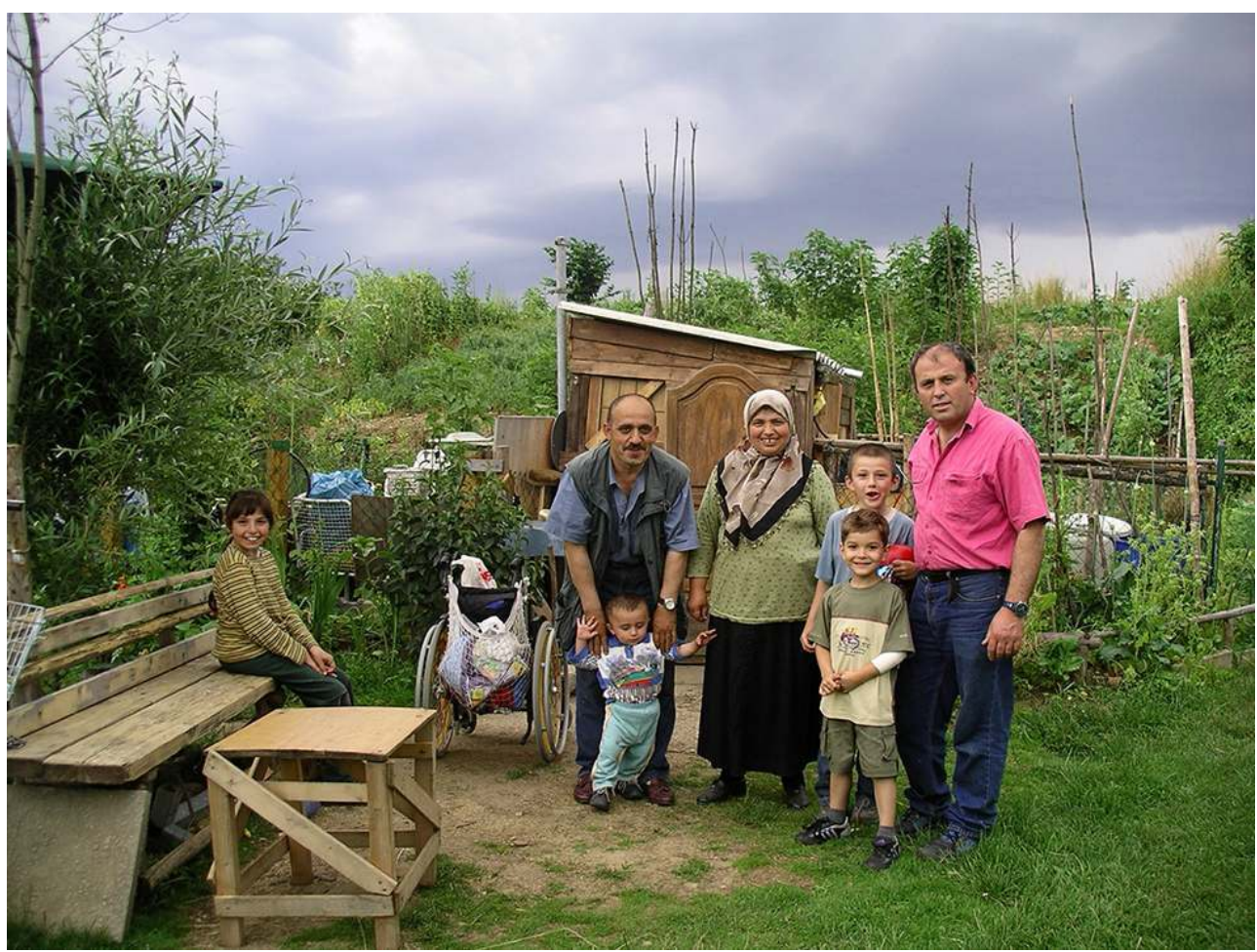

Jardin à Munich - Neuperlach

(c) Konrad Bucher.

La grande majorité des projets de jardins fonctionne en autogestion, mise à disposition gratuite. Il n'y a que de rares cas où des personnes sont employées. Les terrains sont presque toujours mis à disposition par une administration municipale ou une paroisse. Depuis quelques années, des musées, des théâtres et des universités tentent aussi l'expérience des jardins collectifs. Et c'est là que l'on voit particulièrement bien le caractère semi-public du jardin collectif : il a une fonction de passerelle et fait dialoguer les frontières invisibles entre la soi-disant « culture élitiste » et les ressources culturelles des participants ${ }^{3}$. 
5 Même si bien des jardins partagés urbains ont aujourd'hui une dimension inter et transculturelle, l'objectif des Jardins Interculturels est de rassembler des personnes de toutes origines et de mettre volontairement l'accent sur les différences culturelles. Ici, les participants renégocient chaque jour leur propre réalité avec celle des autres. Le quotidien n'est bien sûr pas toujours exempt de conflits mais la conception démocratique de cet espace social fournit des règles claires, par exemple en stipulant, dans les statuts de l'association, que personne ne doit être discriminé du fait de son sexe ou de ses origines. Tous peuvent se prévaloir des statuts de l'association, qui fournissent un cadre au vivre ensemble. La forme d'organisation d'une association à but non lucratif, outre son statut, offre d'autres avantages. Elle permet de recevoir des dons, de conclure des baux et des contrats d'assurance, mais surtout, elle établit une structure démocratique qui permet à tous de s'impliquer et de participer aux décisions.

6 Le « prototype » des Jardins Interculturels s'est développé à Göttingen, ville universitaire du nord de l'Allemagne. En 1995, des femmes bosniaques réfugiées là se réunissent dans le centre d'aide pour les migrants. Elles boivent du thé, confectionnent des ornements de table et attendent la fin de la guerre dans leur pays. La travailleuse sociale du centre d'immigration réfléchit à la façon dont elle pourrait éveiller leur intérêt. Et elle leur pose une question, qui eut de nombreuses conséquences : «Qu'est-ce qui vous manque le plus, ici, en Allemagne? » La réponse des femmes est unanime : « Nos jardins ». Elles racontent que chez elles, elles avaient de grands potagers, avec lesquels elles nourrissaient leur famille. En exil, elles se voient condamnées à l'attente et à l'oisiveté4. La travailleuse sociale saisit aussitôt l'occasion et, ensemble, elles se mettent à la recherche d'un terrain approprié. Les femmes se réjouissent de pouvoir reprendre en main de façon autonome une partie importante de leur vie quotidienne. Un an plus tard, elles signent le premier bail. Un groupe de femmes et d'hommes bosniaques, iraniens, allemands, kurdes, irakiens, éthiopiens et afghans commence à jardiner. 
Figure 2

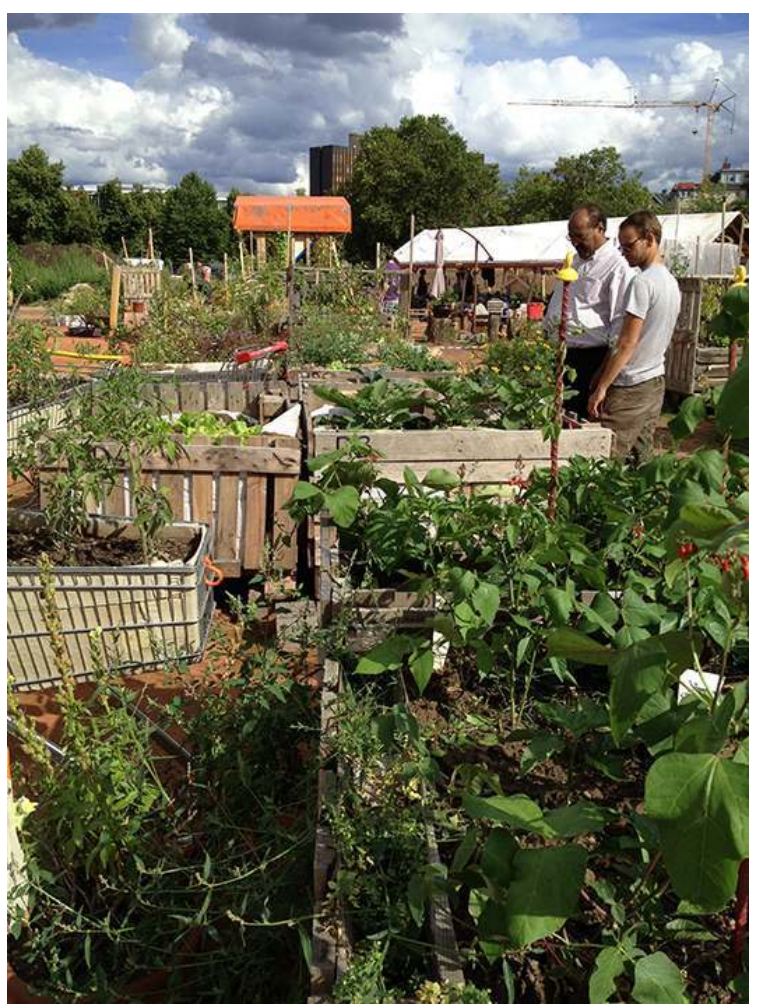

Jardin NeuLand à Cologne. Tassew Shimeles donnant des conseils au jardinier.

(c) Christa Müller.

Tassew Shimeles, l'ingénieur agronome éthiopien-allemand que le centre d'immigration embauche pour diriger le projet, poursuit un plan ambitieux : avec la germano-kurde Najeha Abid, fondatrice de l'association, ils développent, à partir de la pratique, le concept de " jardins internationaux » fondé sur une approche transculturelle (fig. 2). Ici le fait de jardiner ensemble sert de point de départ à des échanges entre personnes de cultures différentes. La compréhension des différences culturelles qui s'exprime dans la relation à la nature et dans les pratiques culturales de chacun a permis de trouver un langage commun. Ce que Shimeles appelle «la langue verte des peuples».

Si l'obligation d'exclure tout engrais chimique ou pesticide prévaut partout, on observe une grande diversité de techniques agricoles dans l'exploitation du sol. Des expérimentations ont lieu sur les parcelles. La plupart des personnes essaient de se procurer des semences et des plantes de leur pays d'origine. Souvent, les jardiniers constatent que les plantes, malgré des conditions bioclimatiques différentes, se développent, s'adaptent à leur nouvel environnement et prennent parfois une nouvelle forme. Mais elles ont bien le même parfum que dans les souvenirs d'enfance de celle qui les cultive. Retrouver l'aspect des plantes et leur parfum réveille des souvenirs biographiques, qui peuvent être "retissés». Les jardins ne sont plus seulement des refuges mais des points d'ancrage biographiques importants. 


\section{Le rôle de la fondation anstiftung}

La fondation anstiftung ${ }^{5}$, dont le siège se trouve à Munich, a accompagné et soutenu l'association des Jardins internationaux de Göttingen depuis sa création. En tant que fondation reconnue d'utilité publique, anstiftung associe une activité de recherche à la mise en réseau des acteurs et au soutien financier des actions. Cette politique résulte d'un investissement de long terme et d'une connaissance approfondie des projets financés ainsi que de leurs méthodologies respectives. L'équipe de recherche d'anstiftung maintient des liens constants avec le terrain, qui comprend actuellement trois réseaux sur l'ensemble du territoire allemand: les Repair Cafés, les Ateliers Partagés (Open Worshops) et le mouvement récent du jardinage urbain qui comprend les jardins partagés (Urban Gardening) ${ }^{6}$.

10 Anstiftung entend étudier et comprendre dans une démarche sociologique les spécificités de chaque projet, refléter et répercuter leur impulsion et leur expertise. Elle est un partenaire qui donne une résonance aux projets et apprend avec eux, grâce à des rencontres de réseau, des webinars (séminaire par internet), des conférences et des ateliers, à l'échelon fédéral ou local. On y échange et donne des informations pratiques et utiles à propos des règles et des conventions avec les administrations, assurances, la communication avec les administrations publiques et la gestion des relations avec le monde de la recherche.

Figure 3

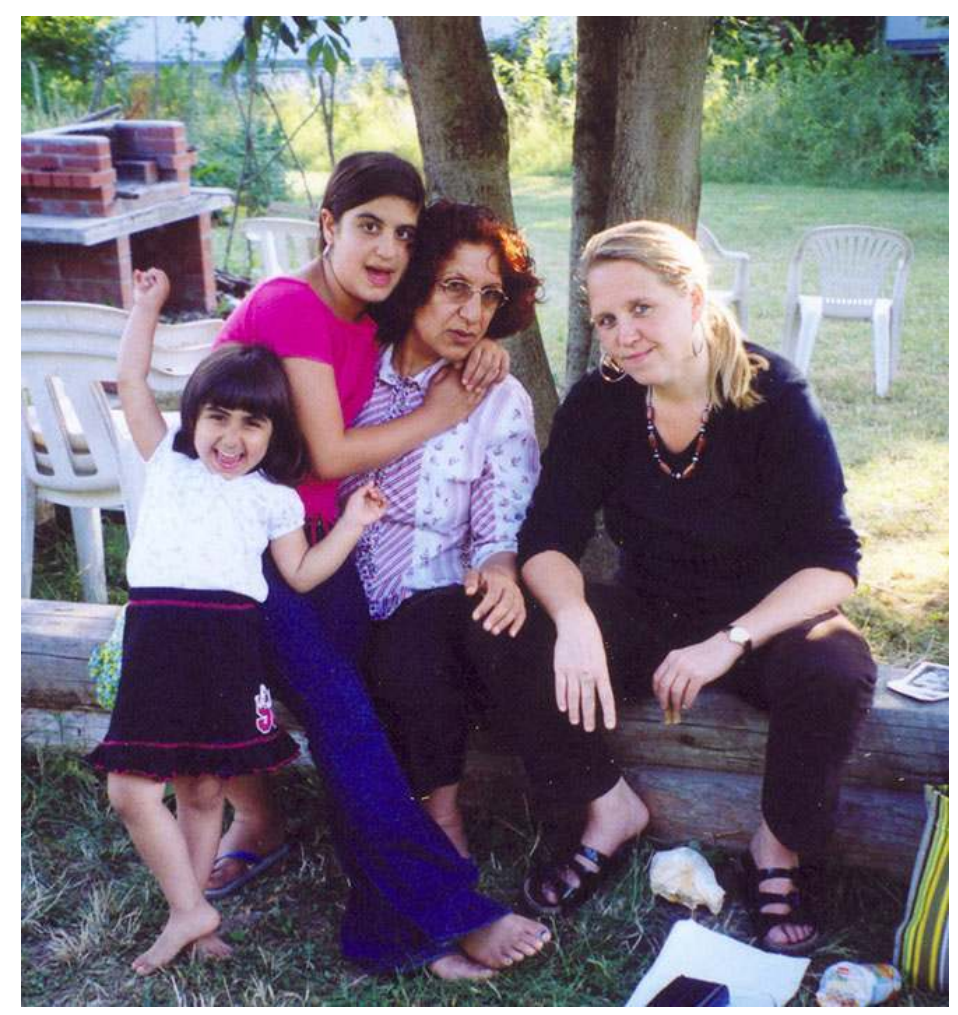

Les Jardins Internationaux de Göttingen avec Christa Müller.

(C) Fondation anstiftung. 
11 J'ai moi-même, dans le cadre de mes activités de recherche auprès d'anstiftung, mené une étude de terrain entre 1999 et 2001 à Göttingen, publiée en 2002 sous le titre Wurzeln schlagen in der Fremde. Die Internationalen Gärten und ihre Bedeutung für Integrationsprozesse ${ }^{7}$ ( Prendre racine en terre étrangère. Les Jardins internationaux et leur importance dans les processus d'intégration »). Après la parution de mon livre, l'intérêt pour le projet s'est accru brusquement: de nombreuses paroisses, municipalités, centres d'accueil de migrants et acteurs individuels de toute l'Allemagne ont voulu savoir quelles étaient les étapes nécessaires pour créer un jardin interculturel (fig. 3). L'association des Jardins internationaux, dirigée par des bénévoles, a vite été dépassée par le flot de questions. Anstiftung a entrepris, en étroite coopération avec l'association de Göttingen, la construction d'un réseau qui s'est peu à peu étendu au territoire national. Il s'est formé en 2003 lors des premières rencontres fédérales du réseau, à Berlin. À l'époque, le réseau réunissait les quatre premiers projets de jardins ; début 2018, anstiftung recensait dans sa base de données 650 jardins collectifs urbains, dont la moitié est explicitement constituée de jardins interculturels ${ }^{8}$. Des associations de jardins interculturels sont nées également en Autriche (2007) et en Suisse (2009). Elles ont bénéficié des conseils d'anstiftung à leurs débuts.

\section{Vecteur de reconnaissance sociale}

12 Dans un jardin interculturel, les acteurs du projet, originaires de nombreux pays, élaborent ensemble chaque jour une nouvelle réalité partagée. Ils déconstruisent par la même occasion une conception idéologique de l'intégration, comprise comme le fait de devenir conforme à la société d'accueil. Cette déconstruction se fait au profit d'une interaction riche en termes d'inclusion et de transformation sociale.

Figure 4

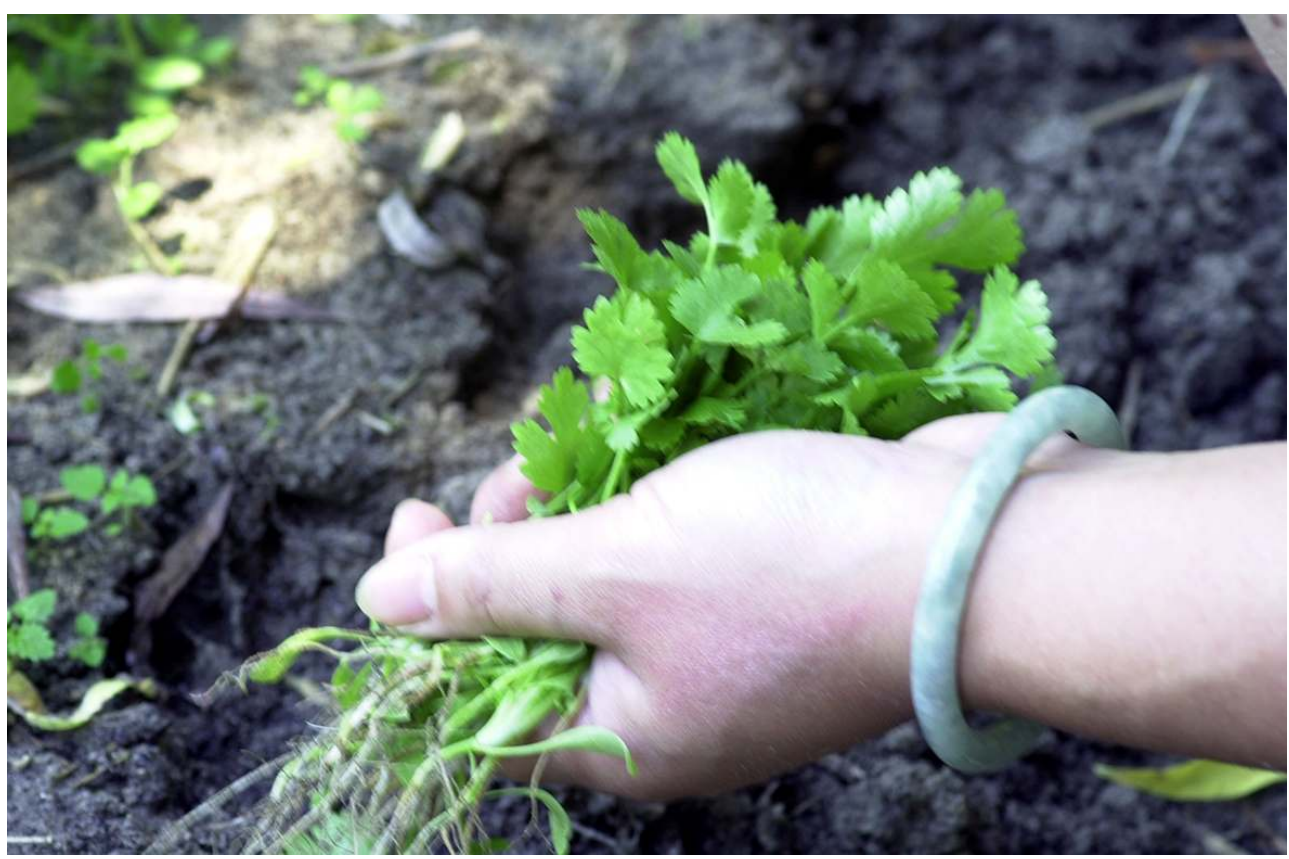

Coriandre.

(C) Cornelia Suhan. 
13 Ce qui différencie les jardins interculturels de nombreuses autres mesures d'intégration, c'est qu'ils sont clairement orientés vers la mise en œuvre et la création de ressources propres. Non seulement les personnes apportent leurs connaissances, mais elles produisent aussi des surplus tangibles, qu'elles peuvent offrir ou échanger (fig. 4). Elles se découvrent productives, donneuses, généreuses et autonomes. Dans le cadre de cette économie du don, les personnes peuvent rencontrer les autres sur un pied d'égalité.

Pour diverses raisons, les jardins interculturels sont les lieux appropriés pour produire de la reconnaissance sociale : ils ne comptabilisent pas les lacunes mais les compétences et les connaissances des participants. Chacun dispose de ressources spécifiques; les migrants, par exemple, apportent leur connaissance du travail de la terre ou des façons de gérer les conflits issues de leur culture. Dans un jardin interculturel, vous faites concrètement l'expérience des potentialités qui se cachent dans des formes culturelles de l'hospitalité, dans de beaux gestes d'inclusion, dans l'art de la narration et dans le souci de l'autre.

Un jardin interculturel propose ainsi des moyens de sortir du « ghetto de l'altérité ». Peu à peu, un engagement civique peut aussi y prendre racine. Quelques acteurs de jardins interculturels siègent aujourd'hui dans les conseils d'intégration des villes, discutent de la sensibilisation des établissements de santé à la dimension interculturelle ou fondent des associations d'entraide sur le thème de la santé ou de la nutrition'.

Aucun jardin, cependant, ne fonctionne «juste comme ça »; ce ne sont pas seulement différentes langues qui s'y rencontrent, mais aussi divers milieux socio-culturels. Tous les projets ont pour ambition de découvrir ce qui est commun au groupe de jardiniers malgré les nombreuses différences. Le cadre facilite la réalisation de cette ambition: gérer ensemble un bout de terrain, voir pousser sa production et être en relation avec d'autres, échanger sur la croissance des plantes ou sur ses propres progrès ou reculs, ce sont les grandes et petites possibilités qu'offre un jardin ${ }^{10}$ pour créer du partage.

Parmi ces possibilités, il y a celle de procurer aux membres des jardins collectifs de quartiers urbains moins privilégiés, un contact avec la nature. La question de la justice environnementale est très liée à celle de la justice sociale. L'une et l'autre ne sont d'ailleurs pas particulièrement présentes à l'heure actuelle dans les sociétés européennes. Bien des personnes actives dans les jardins interculturels disposent de faibles revenus, vivent dans des rues très fréquentées et dans des logements exigus, certaines même dans des containers ou dans des hébergements pour les demandeurs d'asile. Des migrants défavorisés, des chômeurs et des travailleurs précaires ont ainsi en commun l'expérience d'une faible reconnaissance sociale ${ }^{11}$. Dans le sillage de la restructuration de l'Étatprovidence, de la différenciation des marchés du travail et d'une politique d'immigration restrictive dans les pays européens, un nombre croissant de personnes se voient privées de l'accès aux ressources matérielles, à la formation mais aussi à la nature. Les jardins interculturels ne remplacent certes pas une politique sociale mais ils peuvent ouvrir de nouvelles portes aux personnes concernées.

Un argument supplémentaire en faveur des jardins interculturels réside dans le fait qu'ils permettent la rencontre entre des personnes d'origines très diverses. Rencontre hautement improbable sinon. Michael J. Sander démontre, avec son concept de "société de loges VIP », que la rencontre entre personnes d'origines sociales diverses devient de plus en plus rare dans l'espace public. Le philosophe américain y voit un problème 
fondamental pour la démocratie parce qu'il prive beaucoup de citoyennes et de citoyens de l'expérience de la citoyenneté en tant que participation au monde ${ }^{12}$.

Une dimension de la démocratie vécue qu'il ne faut pas sous-estimer réside dans la coprésence avec d'autres, et à cet égard, les jardins interculturels peuvent être vus comme une contribution innovante dans une société qui instaure une distinction toujours plus forte entre les milieux sociaux.

\section{Pratiques d'appropriation de l'espace}

Pour beaucoup de jardiniers, leur propre parcelle est le point de départ à partir duquel ils peuvent s'approprier peu à peu l'espace semi-public du jardin, et de là, l'environnement plus lointain, sous forme d'excursions, de visites, de coopération et de rencontres de réseaux avec d'autres projets de jardins. Mais avant tout, les jardins deviennent rapidement des espaces productifs dans leur quartier. En règle générale ils ne sont pas clôturés, les transitions entre les parcelles et le quartier sont fluides. Dans de nombreux cas, les habitants du quartier ont été associés depuis le début dans la création du jardin, ou dans d'autres cas, les jardiniers ont peu à peu créé des liens avec le quartier.

Il est évident que le jardin et le voisinage bénéficient l'un de l'autre et que les échanges avec le territoire et les institutions ont pour effet que les jardiniers, qu'ils aient ou non un passé d'immigrant, voient leur quartier d'un œil neuf, c'est-à-dire avec les yeux de celui qui participe de sa transformation. À cet égard, ce ne sont pas seulement les parcelles individuelles qui se transforment, bêchées et toujours replantées, c'est aussi le quartier qui se transforme dans la perception collective. Les jardiniers expérimentent différentes formes de participation: par exemple réussir à faire installer une prise d'eau, aider à l'organisation d'une fête de quartier, faire visiter le jardin en offrant aux visiteurs une petite collation. À travers ces expériences, se crée un réseau de relations proches et lointaines qui produit de la reconnaissance pour les jardiniers. On se rencontre dans la rue, on se salue, on échange quelques mots. Des personnes, qui ont parfois passé de longues périodes dans un univers étroit, restreint à des relations basées sur l'appartenance ethnique, dans des camps de réfugiés, ou encore des chômeurs isolés, deviennent des personnalités du quartier. Quand le maire ou les représentants des organisations de migrants viennent dans le jardin en tant que visiteurs, ils ne sont soudain plus des personnes abstraites pour les jardiniers. Ils se laissent offrir les plats et les gâteaux que les gens ont préparés; les frontières s'effacent, au moins temporairement. 
Figure 5

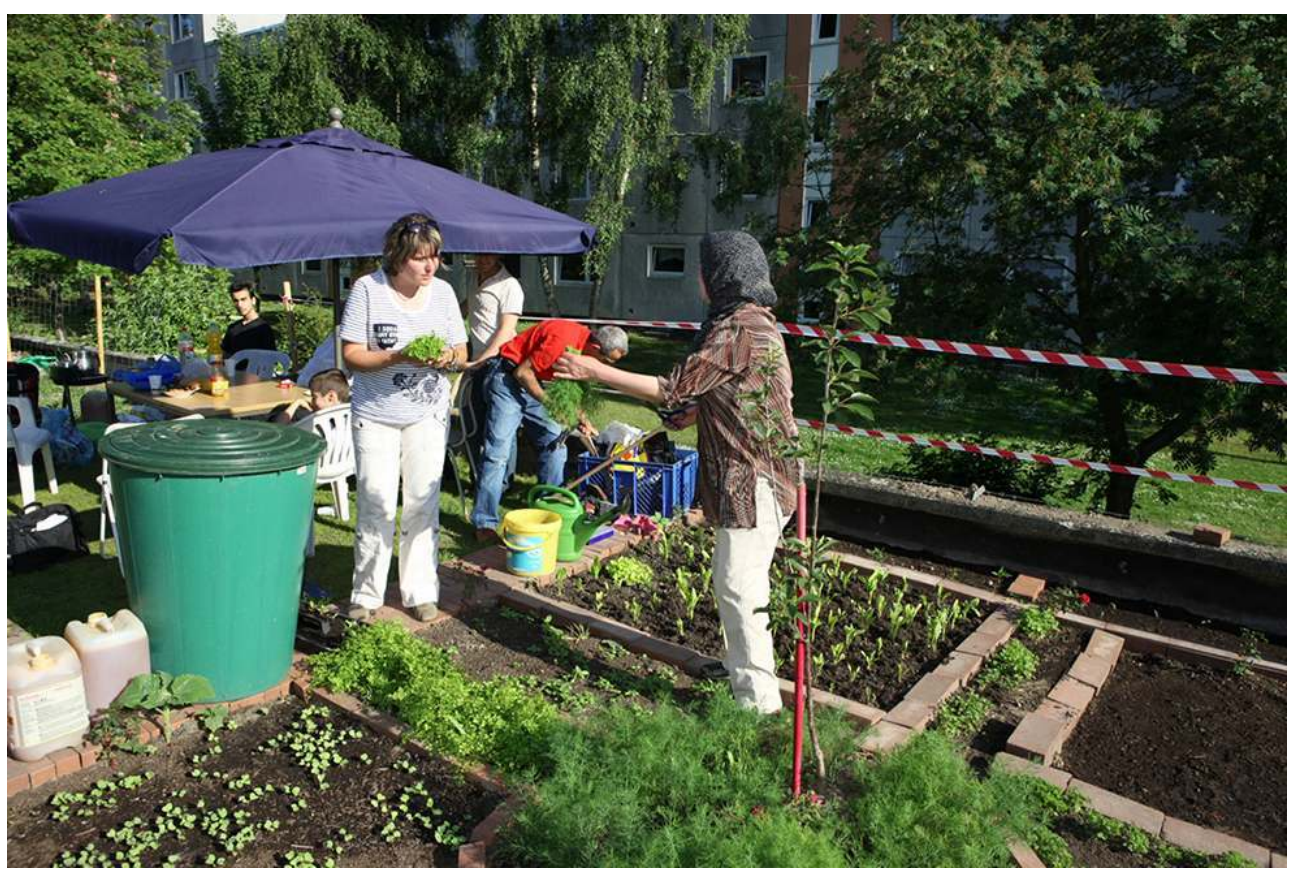

Les Jardins de quartier internationaux à Hanovre.

(c) Cornelia Suhan.

Ce ne sont pas seulement les individus mais l'ensemble de la société urbaine qui en bénéficie parce qu'elle dépend des contributions productives de tous les milieux sociaux pour rester vivante. Dans un documentaire sur les jardins interculturels d'Aalen, une ville du sud de l'Allemagne, une jeune réfugiée d'Azerbaïdjan prend la parole. Elle raconte qu'elle a participé à un concours de photos organisé dans ce jardin. Sa photo d'un escargot a remporté le premier prix. Rayonnante de joie, elle évoque devant la réalisatrice son portrait paru dans le journal, et imagine que les gens se demandent maintenant qui peut bien être cette femme et d'où elle vient: "Beaucoup de gens me connaissent à cause de cette photo. C'est bien pour moi!», dit-elle en souriant ${ }^{13}$. De même un jardinier d'origine indienne, d'âge moyen, témoigne devant la caméra : « Avant, je me sentais étranger. Pas d'endroit où aller. Depuis que j'ai le jardin, je me sens les deux pieds dans le sol. J'ai maintenant un endroit où je peux inviter quelqu'un ${ }^{14}{ }^{\prime}$.

24 À travers les propos de ces deux personnes, on voit bien que la production de capital symbolique revêt une importance centrale dans les jardins interculturels. Anna Mittelsten Scheid constate, dans son enquête qualitative sur le «Jardin de Culture » de Lunebourg (Basse-Saxe), que pour beaucoup, le jardinage n'est pas du tout la motivation principale pour participer au projet. Dans son analyse, la chercheuse en sciences de l'environnement, a pu montrer que les termes «jardinage » et " plantes », dans les récits des participants, n'étaient cités que 48 fois, mais les termes «ensemble» et «en commun » l'ont été 93 fois, et « langue » et « parler », 90 fois ${ }^{15}$. 


\section{Processus transculturels}

25

productif. Le fait de pourvoir eux-mêmes à leurs besoins procure aux participants des jardins interculturels, une autonomie et une estime de soi qui leur permet de rencontrer les autres comme des égaux. Les jardiniers et jardinières ont en main quelque chose : ce qu'ils ont récolté ou produit eux-mêmes, qu'ils offrent ou qu'ils invitent à partager. Cela leur permet de se relier à leur propre culture de l'hospitalité au quotidien. Pouvoir donner quelque chose, cela veut dire, par exemple, pour les réfugiés, changer de regard et sortir d'un statut qui tend à les réduire à des personnes disposant de faibles ressources.

\section{Figure 6}

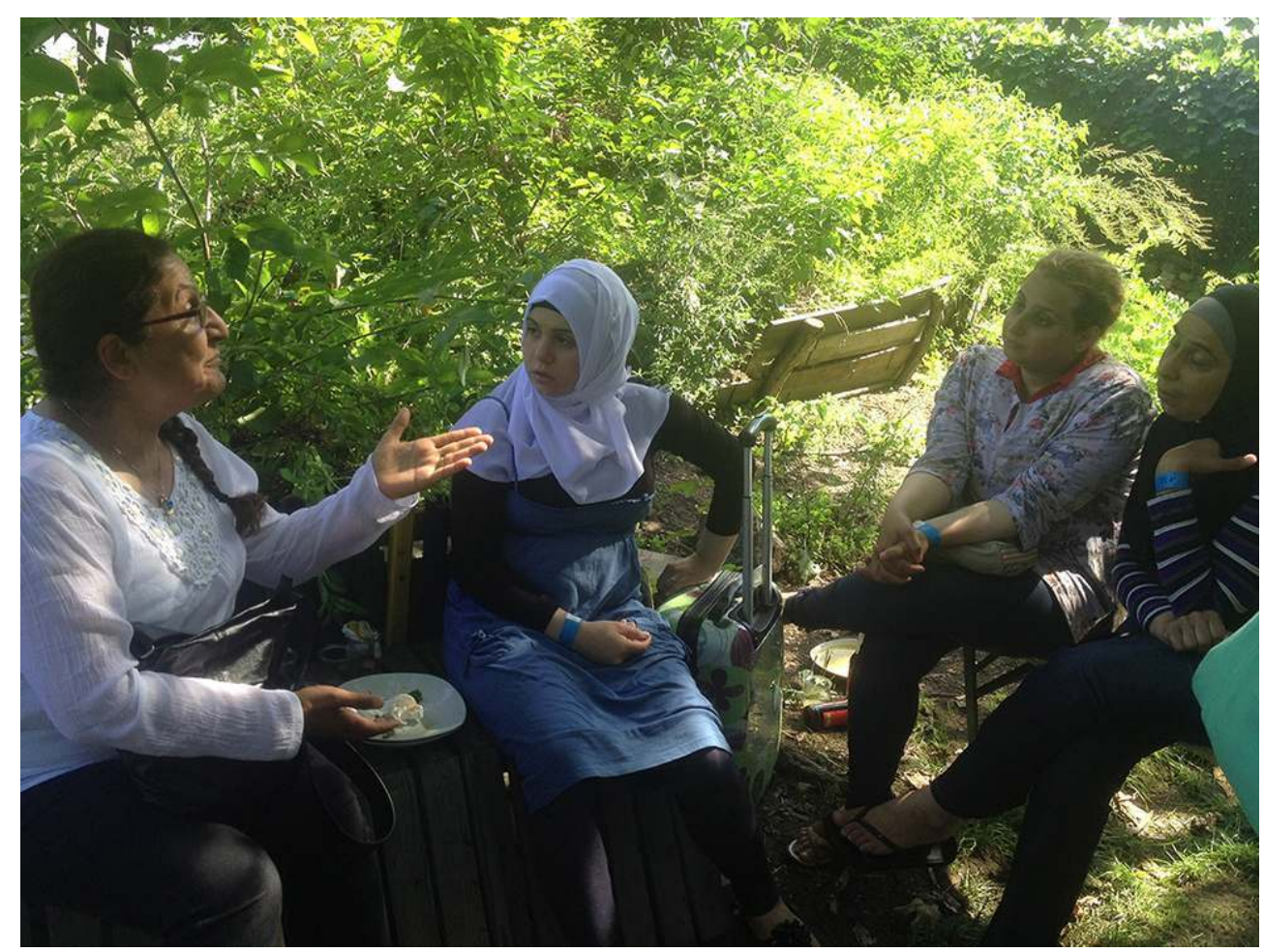

Jardin AnnaLinde à Leipzig avec Najeha Abid.

(c) Christa Müller.

Beaucoup de ces jardiniers sont perçus par la majeure partie de la société allemande comme des résidents temporaires. Dans les jardins interculturels, ils ne ressentent pas leur présence comme seulement «tolérée », au contraire, ils peuvent même être là ceux qui reçoivent les autres (fig. 6). Être privé d'un emploi et être considéré comme bénéficiaire à vie d'aides sociales représente, pour beaucoup de migrants, une expérience renouvelée de l'exclusion. Les femmes subissent fréquemment une double exclusion, à 
travers les assignations sociales et les violences réelles : leur capacité de mouvement est limitée, elles ne se retrouvent pas dans des lieux publics mais dans des appartements exigus. Les jardins, eux, sont situés au-delà des représentations de l'espace privé et de l'espace public propres à chaque culture. Begzada Alatovic, fondatrice du jardin des femmes « Rosenduft » («Parfum de roses ») à Berlin, décrit cette dimension essentielle au cours d'un entretien que je fis avec elle :

«Les femmes restent toujours chez elles, font la cuisine, des gâteaux, ne parlent souvent pas un mot d'allemand, n'ont aucune formation professionnelle, pas de permis de travail, alors le jardin est pour elles la seule possibilité de sortir, d'être tout simplement avec d'autres personnes [...]. J'ai vu des femmes qui avaient passé plusieurs années dans un camp de réfugiés à Srebrenica, et qui ici, lorsque le premier oignon est sorti de terre, étaient émerveillées! Elles parlent aussi avec les plantes, c'est une relation spéciale. Et elles ont aussi un grand savoir, sans être jamais allées à l'école. Elles peuvent le transmettre ici, au jardin, et en parler. C'est mieux que de rester chez soi pour ne parler que de maladie et de guerre ${ }^{16}{ }^{\prime}$.

Les raisons de la grande réussite des jardins interculturels concernant l'intégration sont à chercher avant tout dans leur positionnement spécifique, dans des «interstices »; à la différence de nombreux autres projets d'intégration, ces jardins construisent à bien des égards une transition entre pays d'origine et pays d'accueil ainsi qu'entre le passé et le présent des jardiniers ${ }^{17}$.

Dans les jardins interculturels, les participants ont l'occasion de réfléchir au quotidien à la fois sur leur propre culture et à la fois sur leur relation à d'autres pratiques culturelles. Les jardiniers construisent ainsi « une carte de l'interculturalité » où ils peuvent tous trouver leur place.

Que cela constitue une alternative intelligente à l'argument simpliste du concept de culture dominante, c'est une évidence.

\section{NOTES}

1. - Docteure en sociologie, Christa Muller, par ses recherches, a accompagné dès le début le mouvement de l'Urban Gardening et ses premières manifestations sous la forme de jardins interculturels en Allemagne. Elle est la directrice de la fondation anstiftung, qui soutient et étudie le mouvement des jardins urbains en Allemagne en tant qu'organisme de recherche et financeur. Voir le site : www.anstiftung.de. [consulté le 05/12/2018].

2. - CHERVEL, Thierry, SEELIGER, Anja (éd.). Islam in Europa. Eine internationale Debatte. Francfortsur-le-Main : Suhrkamp, 2007.

3. - KIEFER, Theresia, ZECHLIN, René, WILHELM HACK MUSEUM (éd.). Ein Garten für alle! Museum trifft Urban Gardening. Fribourg : Orange Press, 2017.

4. - MÜLLER, Christa. Wurzeln schlagen in der Fremde. Die Internationalen Gärten und ihre Bedeutung für Integrationsprozesse. Munich : Ökom, 2002, p. 16.

5. - NdT : le mot allemand Anstiftung veut dire «incitation». La fondation a choisi d'écrire son nom sans majuscule.

6. - MÜLLER, Christa (éd.). Urban Gardening. Über die Rückkehr der Gärten in die Stadt. Munich : Ökom, 2011. 
7. - MÜLLER, Christa. Wurzeln schlagen in der Fremde. Op. cit.

8. - Voir le site: https://anstiftung.de/urbane-gaerten/gaerten-im-ueberblick [consulté le 05/12/2018].

9. - BAIER, Andrea. Wie soll man gesund sein, wenn man keine Arbeit hat? Gesundheit und soziale Ungleichheit. Erfahrungen einer Frauengruppe mit einem Gesundheitsprojekt. Bielefeld: Transcript, 2013.

10. - WERNER, Karin. «Interkulturelle Gärten als Sozialräume der Mikro-Integration ». Skripte zu Integration und Nachhaltigkeit, $\mathrm{n}^{\circ} 6,2008$. Voir sur le site: https://anstiftung.de/jdownloads/ Skripte\%20zu\%20Migration\%20und\%20Nachhaltigkeit/Skript6_Mikro-Integration.pdf [consulté le 05/12/2018].

11. - HEITMEYER, Wilhelm. Deutsche Zustände. Folge 10. Berlin/Francfort-sur-le-Main : Suhrkamp, 2010.

12. - SANDEL, Michael J. Was man für Geld nicht kaufen kann. Die moralischen Grenzen des Marktes. Berlin : Ullstein, 2012 (trad. de : What money can't buy, Mcmillan 2012 ; trad. fr. : Ce que l'argent ne saurait acheter. Les limites morales du marché. Trad. Christian Cler, Paris : Seuil, 2014).

13. - HAIDE, Ella von der. Eine andere Welt ist pflanzbar, Teil 5. Urbane Gemeinschaftsgärten in Deutschland. DVD, 55 min. Munich : anstiftung 2016, min. 06.10.

14. - Ibid. $\min 07.03$.

15. - MITTELSTEN SCHEID, Anna. "Langsam, langsam also sind wir also eine Familie, die Kulturgarten heißt" - Wie die Forderung nach Integration von Teilnehmer*innen eines interkulturellen Gartenprojekts in Lüneburg verhandelt wird. Leuphana Universität Lüneburg: Bachelorarbeit, 2017, p. 24.

16. - BAIER, Andrea. Op. cit., p. 12 sq.

17. - WERNER, Karin. Art. cit.

\section{RÉSUMÉS}

Le mouvement d'exode déclenché par la guerre civile en Syrie a ravivé le débat public sur le concept, toujours flou, de « culture dominante ». Comme on l'a vu dans les récents affrontements entre partisans du multiculturalisme et tenants d'une exigence stricte d' "assimilation» des immigrés dans une société d'accueil que l'on imagine statique, on constate actuellement une aggravation de ce dualisme. Mais on exclut souvent de ces discussions les réalités concrètes de la vie des migrantes et migrants. Dès le mouvement d'exil entraîné par la guerre de Bosnie, au milieu des années 1990, est né un projet innovant qui a expérimenté une démarche constructive vis-à-vis des différences culturelles, et que l'on peut voir comme une réponse pratique aux exacerbations des tensions sous-jacentes. Dans le projet des jardins interculturels, le fait de jardiner ensemble donne naissance à des processus transculturels de rencontre et d'échange. Dans ces jardins collectifs, on utilise les ressources qu'apportent de leurs pays d'origine ces hommes et ces femmes comme point de départ d'un processus d'autonomisation, et ce, de façon constructive. Cette contribution montre le développement des jardins interculturels depuis 1996 à travers l'exemple des «Jardins Internationaux de Göttingen» et traite des différentes potentialités de cette innovation sociale.

The massive exodus provoked by the civil war in Syria has breathed new virulence into the public debate around the rather ill-defined concept of 'dominant culture'. In the recent clashes between the proponents of multiculturalism and the supporters of a strict requirement of 'assimilating' 
immigrants into the society which welcomes them-always seen as something stable-there is an aggravation of this dualism. But these debates often leave out the concrete realities of the lives of immigrant men and women. At the time of the movement which exiled many from Bosnia, during the war there in the mid-1990s, an innovative project was developed which tried out a constructive approach to the question of cultural differences, and which can be seen as a practical answer to the exacerbation of underlying tensions. In this project of intercultural gardens, the act of gardening together gives rise to transcultural processes of meeting and exchange. In these collective gardens, the resources brought from their country of origin by immigrant men and women serve as a point of departure for a process of empowerment, in a constructive way. This contribution examines the development of intercultural gardens since 1996, through the example of the 'International Gardens of Göttingen' and looks at the different possibilities of this social innovation.

INDEX

Mots-clés : jardin interculturel, jardinage urbain, réfugiés, intégration, interculturalité, inégalité sociale, autarcie urbaine

Keywords : intercultural garden, urban gardening, refugees, integration, interculturality, social inequality, urban self-reliance

\section{AUTEUR}

\section{CHRISTA MÜLLER}

Docteure en sociologie www.anstiftung.de christa.mueller@anstiftung.de 